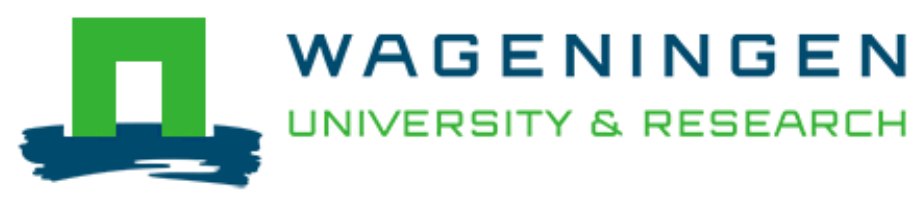

Tiny but mighty: bacterial membrane vesicles in food biotechnological applications

Liu, Y., Alexeeva, S., Defourny, K. A. Y., Smid, E. J., \& Abee, T.

This is a "Post-Print" accepted manuscript, which has been published in "Current Opinion in Biotechnology"

This version is distributed under a non-commercial no derivatives Creative Commons (C) $(1) \Theta($ (CC-BY-NC-ND) user license, which permits use, distribution, and reproduction in any medium, provided the original work is properly cited and not used for commercial purposes. Further, the restriction applies that if you remix, transform, or build upon the material, you may not distribute the modified material.

Please cite this publication as follows:

Liu, Y., Alexeeva, S., Defourny, K. A. Y., Smid, E. J., \& Abee, T. (2018). Tiny but mighty: bacterial membrane vesicles in food biotechnological applications. Current Opinion in Biotechnology, 49, 179-184. DOI: 10.1016/j.copbio.2017.09.001

You can download the published version at:

https://doi.org/10.1016/j.copbio.2017.09.001 


\title{
Tiny but mighty: bacterial membrane vesicles in food biotechnological applications
}

\author{
Yue Liu, Svetlana Alexeeva, Kyra A.Y. Defourny, Eddy J. Smid*, Tjakko Abee
}

Address: Wageningen University, Laboratory of Food Microbiology, P.O. Box 17, 6700 AA Wageningen, the Netherlands

*) Corresponding author: Eddy J. Smid (eddy.smid@wur.nl)

\begin{abstract}
Membrane vesicle (MV) production is observed in all domains of life. Evidence of MV production accumulated in recent years among bacterial species involved in fermentation processes. These studies revealed MV composition, biological functions and properties, which made us recognize the potential of MVs in food applications as delivery vehicles of various compounds to other bacteria or the human host. Moreover, MV producing strains can deliver benefits as probiotics or starters in fermentation processes. Next to the natural production of MVs, we also highlight possible methods for artificial generation of bacterial MVs and cargo loading to enhance their applicability. We believe that a more in-depth understanding of bacterial MVs opens new avenues for their exploitation in biotechnological applications.
\end{abstract}

\section{I ntroduction}

Production of extracellular membrane vesicles (MVs or EVs) is a conserved phenomenon in Eukarya, Archaea and Bacteria $\left[1^{\circ} 2^{\bullet}\right.$. Being lumen-containing spheres enclosed by lipid-bilayers, MVs are identified in different sizes ranging from $20 \mathrm{~nm}$ to $500 \mathrm{~nm}$ in diameter, and with various cargo components, including proteins, DNA, RNA, signalling molecules, etc. Moreover, MVs are considered to have essential functions in cellular life, i.e., cell-to-cell communication, competition, survival or stress response of cells $\left[3^{\bullet}-6\right]$.

In bacteria, MV production was first observed in Gram-negatives over fifty years ago, and there have been an increasing number of studies performed on these MVs in recent decades [2*]. Gram-negative bacterial MVs are often referred to as outer-membrane vesicles (OMVs), since they are derived from the outer membrane of the bacteria via budding processes [7]. In contrast, due to the presence of a thick cell wall in Gram-positive bacteria, MV production was expected to be absent. This assumption resulted in a three-decade delay in the discovery of Gram-positive MVs compared to their Gram-negative counterparts, although in recent years the evidence of MV production in Gram-positive bacteria accumulated rapidly $\left[2 \bullet, 3^{\bullet}\right]$.

Recent advances in the study of MV production in Gram-positive bacteria are particularly interesting for the food biotechnological field. The Gram-positive lactic acid bacteria (LAB) are key players in various food and feed fermentation processes as starter cultures, probiotics and producers of vitamins [8,9]. Among these, evidence has so far been collected for MV production from Lactococcus lactis (S. Alexeeva et al., unpublished) and Lactobacillus plantarum [10*0. Bacillus subtilis was shown to secrete MVs as well [11], and certain strains of B. subtilis are involved in the production of fermented soybeans known as "natto" while boosting this food product with high levels of vitamin K2 [12]. Among Gram-positive Actinobacteria, species of the genus Bifidobacterium were associated with probiotic effects as well as with MV production [13・•].

To date, comprehensive studies have been mostly focusing on MVs produced by pathogenic bacteria $\left[14^{\bullet}, 15-22\right]$. One of the best-identified functions of MVs is indeed associated with pathogenesis, where they serve as the vehicles for delivering virulence factors and toxins to the host cells $\left[3^{\bullet}, 5,22\right]$. As MVs are able to trigger responses of the host immune system, the application of vaccines against meningitis was realized using OMVs derived from the Gram-negative bacterium Neisseria meningitidis [23]. The potential of vaccine development based on Gram-positive MVs was recognized as well: vaccination with MVs derived from Clostridium perfringens, Streptococcus pneumoniae, Mycobacterium tuberculosis and Staphylococcus aureus were demonstrated to be effective in mice models against infections by respective bacteria [24-27]. However, the possibility of applying microbial MVs in other areas of biotechnology 
remains largely unexplored. In this paper, we provide an overview on current understanding of microbial MVs, with a focus on potential applications of MVs from food-associated bacteria.

\section{Natural functions and biotechnological applications of bacterial MVs}

The composition of various MVs has been studied and has supported the formulation of hypotheses regarding their natural function. Although so far the studies of Gram-positive MVs are not as extensive, the available data already suggest that Gram-positive MVs have similar roles to their Gram-negative counterparts [28]. This knowledge served as a basis for us to propose potential applications of microbial MVs in food biotechnology, in which Gram-positive bacteria are intensively involved (Figure 1).

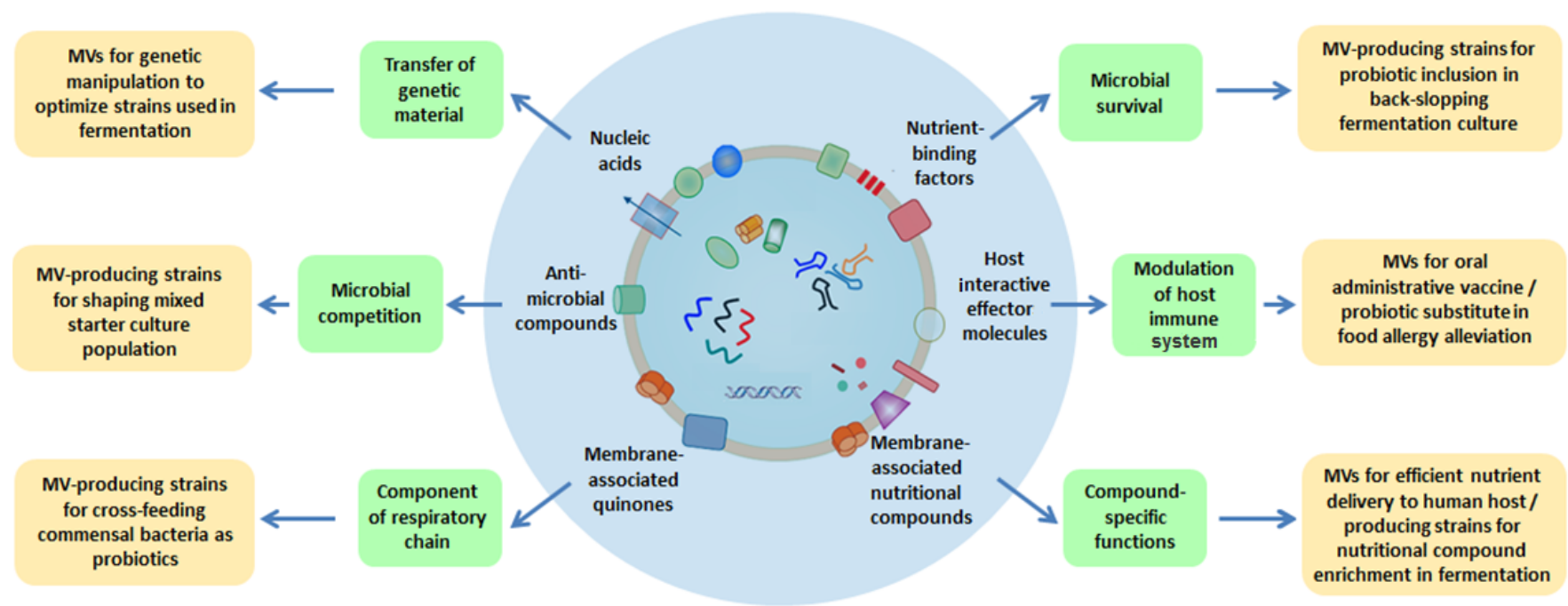

Figure 1. Microbial MV cargos, functions and potential applications. Examples of MV cargos (blue circle), corresponding natural function of the MV carrying the cargo (green box) and proposed applications of the MVs or MV-producing strains (orange box) are presented. MV scheme modified from EVpedia $[29 \bullet \cdot 30]$.

Nucleic acids, including chromosomal DNA, extracellular DNA, plasmids, phage DNA, rRNA, tRNA, mRNA and intragenic RNA species are frequently found as cargo in bacterial MVs (Figure 1) [16,31-34]. MVs protect the nucleic acids from degradation by nucleases and are proposed to facilitate horizontal gene transfer in the microbial community. Gene transfer by MVs within or between species was demonstrated in various genera of Gram-negative bacteria and certain Gram-positive species [35,36]. As only a number of genes were retrieved from the MVs, the incorporation of nucleic acids into MVs might be a selective mechanism instead of a random process [20], although extra assessments are needed for a conclusive statement. In this regard, MVs may provide the opportunity to deliver designed or natural (large) DNA or RNA molecules to recipient bacteria in a controlled manner. Similarly, membrane-derived vesicles have been considered as novel tools for delivering therapeutic genome editing elements (i.e., CRISPR/Cas cassettes) to human cells [37]. Interestingly, natural MV-mediated gene transfer is not considered to result in genetically modified organisms (GMOs) under current legislation (Directive 2001/18/EC [38]), and is therefore especially interesting for food biotechnological applications. MV producing strains with favourable traits could be used to naturally transfer nucleic acids to recipient bacteria, providing them resistance to environmental stresses or bacteriophages, enhanced productivity etc., thus optimizing strains used in starter cultures and other fermentation processes (Figure 1).

In addition to nucleic acids, MVs can also carry other cargo that as well plays a role in microbial competition, survival and fitness increase in a microbial community (Figure 1). Antimicrobial compounds and bacteriolytic enzymes including autolysins and extracellular bacteriolytic enzymes, carried by MVs of particular bacterial species are suggested to exert an inhibitory effect on sensitive co-existing bacteria or fungi $\left[4^{\bullet}, 39,40\right]$. MVs derived from several species were found to contain factors such as iron-binding 
proteins, contributing to increased survival of bacteria under nutrient limiting conditions [41,42]. A few cases also revealed a protective effect of MVs that carry enzymes which degrade certain antimicrobial compounds $\left[3^{\bullet}, 43,44\right]$. This type of protection can also benefit other bacterial species in the same community. Moreover, bacterial cross-feeding through trafficking of membrane-associated compounds, i.e., vitamin K2 (menaquinone), has been observed between L. lactis and another species [45]. In bacteria, menaquinones function as electron carriers in the membrane-embedded respiratory electron transport chain [46]. As a result of menaquinones cross-feeding, the respiratory chain was completed in the recipient bacteria resulting in stimulated growth [45]. Although the cross-feeding mechanism was not completely elucidated in this particular case, MVs may indeed serve as vehicles for microbial crossfeeding of compounds, especially the hydrophobic, conceivably membrane-associated ones.

These ecological roles of MVs highlight the interests of using MV producing bacterial strains in fermentation starter cultures or as probiotics. In stably-maintained, multi-strain starter cultures, strains producing MVs with an inhibitory effect on co-cultured bacteria may suppress the undesirable dominance of one single strain which in term prevents clonal sweeps of strains present at low abundance, thereby ensuring compositional diversity of the starter cultures. Compositional diversity of a microbial community is considered to be the basis of the functional stability (robustness) of the mixed starter culture in face of environmental fluctuations [47]. Moreover, the bacteriolytic enzymes carried by MVs may play a role in bacterial lysis and improved aroma formation during cheese ripening, which adds to the interest of applying MV-producing strains in starter cultures. It may also be desirable to include probiotic strains into well-established fermentation cultures generated by back-slopping. However, newly added strains in such complex microbial communities are often excluded shortly after addition to the culture, a process which is explained by the competitive exclusion principle [48]. The proposed role in competition and survival served by MVs suggests that probiotic strains producing MVs outstanding in these features may establish themselves more easily in complex fermentation cultures. The role of MVs as vehicles for crossfeeding to commensal bacteria also addresses the probiotic value of the MVs and their producing strains, as they may promote the fitness of certain commensals in the gut microbiota.

Besides microbe-microbe interactions, MVs also play a role in the interaction between gut-inhabiting bacteria and their vertebrate host. Bacterial extracellular MVs have been shown to interact with the plasma membrane of host cells and deliver effector molecules (Figure 1) [49,50]. In pathogenic bacteria, these effector molecules are often associated with virulence and can trigger host immune responses. Based on this phenomenon, the potential of developing vaccines from bacterial MVs has indeed been recognized. Moreover, it was demonstrated that MVs produced by the probiotic Bifidobacterium longum can penetrate through intestinal epithelial cells of the host and alleviate food allergy responses in a mouse model $\left[1^{\bullet \bullet}\right.$ ]. The family 5 extracellular solute-protein carried by the MVs selectively induced apoptosis of mast cells without compromising T-cell immune response. Another study showed that L. plantarum-derived MVs up-regulated the expression of host defence genes and provided the host with protection against pathogenic bacteria in Caenorhabditis elegans and human cells [10••]. These findings shed new lights on the extra probiotic value that MVs add to the producing bacteria, and even suggest the possibility of using MVs as a substitute for bacteria to achieve certain probiotic effects. Given the fact that MVs offer protection and effective transport of functional molecules, and are able to penetrate intestinal epithelial cells and subsequently interact with the host immune system, the feasibility to vaccinate human or livestock via oral administration of bacterial MVs seems high. Besides, MVs may also serve as vehicles for efficient delivery of nutritional compounds to the host (Figure 1). For instance, vitamin K2 (menaquinone) is uniquely produced by bacteria and functions in the human body as a carboxylase co-factor for maturation of proteins involved in many vital physiological processes [51]. The natto fermentation bacterium B. subtilis, certain strains of lactic acid bacteria and propionibacteria are among the producers of vitamin K2 $[12,52,53]$, which offers the opportunity for natural vitamin K2 fortification of fermented food products. Menaquinones are hydrophobic compounds which accumulate in the bacterial cell membrane. Although experimental evidence is still required, incorporation of these compounds in the MVs can be speculated. In that case, MVs could have advantages over the whole-cell bacteria in terms of delivering vitamin $\mathrm{K} 2$ to the host, as the intestinal absorption may be achieved without the need to first overcome the bacterial cell envelope barrier and solubilize the vitamin via the lipid digestion route. Therefore, MVs carrying nutritional compounds could be an ideal formulation to include in fermented foods or food supplements to further promote the health benefits.

MVs may indeed incorporate cell membrane-associated compounds that are of interest for human health (e.g. vitamin $\mathrm{K} 2$, certain fatty acids) or for favourable food product traits (e.g. hydrophobic aromatic 
compounds) (Figure 1). In this context, bacterial MV production might provide chances for in situ enrichment of fermented foods or for biotechnological production methods.

\section{Methods of bacterial MV production}

With many potential applications of bacterial MVs proposed, efficient production of MVs with the desired functionality could be one of the major challenges in practice. Indeed, isolation and characterization of MV producing bacterial strains, understanding of the mechanisms governing natural MV production, identification of MV components and interpretation of MV functions are prerequisites to successful applications of naturally secreted bacterial MVs or the producing strains (Figure 2A). Studies have been performed to reveal conditions that encourage the secretion of MVs from Gram-negative bacteria [22,54], and the influencing factors for MV production from Gram-positive bacteria are being recognized gradually $[11,17,28]$. However, it should be realized that artificial MV production evades the limitations determined by the biological properties of bacteria in terms of MV yield and opens doors to even more opportunities (Figure 2B-D). Artificial MV production discussed in this context can be achieved at different levels: the artificial creation of bacterial cell-derived vesicles and the artificial loading of functional compounds into the MVs. Cell disruption due to osmotic pressure (after enzymatic removal of cell walls) or mechanical force (treatment with French pressure cell or sonication) [55] generates membrane fragments that spontaneously form vesicles by hydrophobic interaction. When the desired compounds are naturally accumulated in the bacterial cell membrane or intracellular space, artificial MVs can be directly collected after cell disruption: the membrane associated compounds are carried by the vesicle membrane, and the intracellular compounds are engulfed in the lumen (Figure 2B). When the desired compounds are not naturally present in the bacteria, artificial loading of membrane-associated or lumenal compounds in addition to cell disruption is required to generate the MV with desired effect (Figure 2C and D). Notably, different compounds can be loaded in combination to the MVs to achieve synergy. 

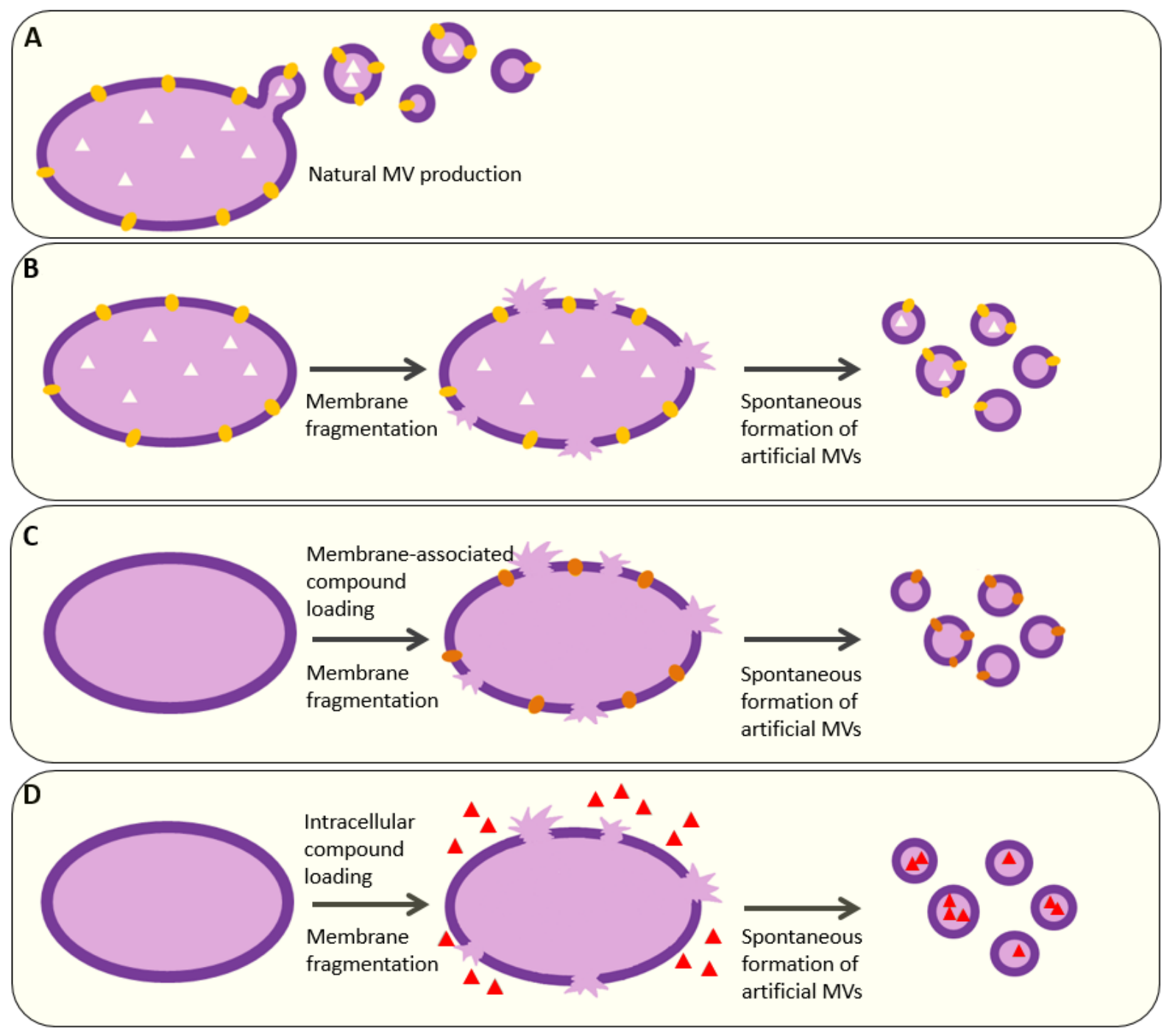

Figure 2. Scheme of different MV production methods. A) Natural MVs produced by bacteria with desired lumenal cargos (white triangles) or membrane-associated cargos (yellow ovals). B) Artificial MVs produced by fragmenting bacteria with desired lumenal or membrane-associated cargos. C) Artificial MVs produced by fragmenting bacteria and artificially loading membrane-associated cargos (orange ovals). When hydrophobic compounds are added to the biomass, they adsorb or incorporate into the membrane. D) Artificial MVs produced by fragmenting bacteria and artificially loading lumenal cargos (red triangles). When water-soluble compounds are added to the biomass, they get encapsulated upon resealing of the membrane fragments. Bacterial cell membrane is depicted as thick purple line.

\section{Concluding remarks}

In this opinion paper, we propose diverse applications of bacterial MVs in the field of food biotechnology based on their natural functions or properties. These applications include genetic engineering tools for strain optimization, agents for shaping starter culture communities, substitutes for probiotics, orally administered vaccines, delivery vehicles for nutritional compounds to the host and the use of natural enrichment of valuable membrane-associated compounds in fermented foods or food supplements. Future applications will also profit from the information contained on EVpedia, a community web source for prokaryotic and eukaryotic extracellular vesicles research $\left[29^{\bullet \bullet}, 30\right]$. We foresee a bright future for applications of these tiny, but mighty, nano-sized MVs. 


\section{References and recommended reading}

Papers of particular interest have been highlighted as:

- of special interest

$\bullet$ of outstanding interest

1. Deatherage BL, Cookson BT: Membrane vesicle velease in bacteria, eukaryotes, and archaea: a conserved yet underappreciated aspect of microbial life. Infect. Immun. 2012, 80:1948-1957.

2. $\quad$ Brown L, Wolf JM, Prados-Rosales R, Casadevall A: Through the wall: extracellular vesicles in Gram-positive bacteria, mycobacteria and fungi. Nat Rev Microbiol. 2015, 13:620-630.

This review presents the current status and draws attention to studies into extracellular MVs from microorganisms with thick cell walls, and discusses hypothetical release mechanisms, cargos and functions associated with MVs from these species.

3. - Kim JH, Lee J, Park J, Gho YS: Gram-negative and Gram-positive bacterial extracellular vesicles. Semin. Cell Dev. Biol. 2015, 40:97-104.

This review provides an overview on the Gram-negative and Gram-positive bacterial extracellular MVs, with a focus on their physiological and pathological functions in both bacteria-bacteria and bacteria-host interactions.

4. Yáñez-Mó M, Siljander PR-M, Andreu Z, Zavec AB, Borràs FE, Buzas EI, Buzas K, Casal E, Cappello F, Carvalho J, et al.: Biological properties of extracellular vesicles and their physiological functions. J. Extracell. vesicles 2015 http://dx.doi.org/10.3402/jev.v4.27066

5. Avila-Calderón ED, Araiza-Villanueva MG, Cancino-Diaz JC, López-Villegas EO, Sriranganathan N, Boyle SM, ContrerasRodríguez A: Roles of bacterial membrane vesicles. Arch Microbiol 2015, 197:1-10.

6. Turturici G, Tinnirello R, Sconzo G, Geraci F: Extracellular membrane vesicles as a mechanism of cell-to-cell communication : advantages and disadvantages. Am J Physiol Cell Physiol 2014, 306:C621-C633.

7. Schwechheimer C, Kuehn MJ: Outer-membrane vesicles from Gram-negative bacteria: biogenesis and functions. Nat Rev Microbiol. 2015, 13:605-619.

8. Hugenholtz J, Smid EJ: Nutraceutical production with food-grade microorganisms. Curr. Opin. Biotechnol. 2002, 13:497-507.

9. Shah N, Patel A: Recent advances in biosynthesis of vitamin and enzyme from food grade bacteria. Intl.J.Food.Ferment.Technol. 2014, 4:79-85.

10. • Li M, Lee K, Hsu M, Nau G, Mylonakis E, Ramratnam B: Lactobacillus-derived extracellular vesicles enhance host immune responses against vancomycin-resistant enterococci. BMC Microbiol. 2017 https://doi.org/10.1186/s12866-017-0977-7

This study demonstrates that extracellular MVs produced from Lactobacillus plantarum up-regulate the expression of host defense genes and provide protection against vancomycin-resistant enterococci infection, highlighting the probiotic effects delivered by MVs and the potential to develop therapeutic treatment of antimicrobial resistant infections by using EVs derived from probiotic bacteria.

11. Brown L, Kessler A, Cabezas-Sanchez P, Luque-Garcia JL, Casadevall A: Extracellular vesicles produced by the Grampositive bacterium Bacillus subtilis are disrupted by the lipopeptide surfactin. Mol Microbiol. 2014, 93:183-198.

12. Sato T, Yamada Y, Ohtani Y, Mitsui N, Murasawa H, Araki S: Efficient production of menaquinone (vitamin K2) by a menadione-resistant mutant of Bacillus subtilis. J. Ind. Microbiol. Biotechnol. 2001, 26:115-120.

13. •• Kim J-H, Jeun E-J, Hong C-P, Kim S-H, Jang MS, Lee E-J, Moon SJ, Yun CH, Im S-H, Jeong S-G, et al.: Extracellular vesiclederived protein from Bifidobacterium longum alleviates food allergy through mast cell suppression. J Allergy Clin Immunol 2016, 137:507-516.

This study provides supporting evidence of the mechanism that extracellular MVs from Bifidobacterium longum can penetrate through intestinal epithelial cells and induce apoptosis of mast cell selectively and consequently alleviate food allergys symptoms. It stresses the probiotic effect delivered by bacterial MVs and the potential applications of MVs in treating allergic diseases.

14. - Haas B, Grenier D: Isolation, characterization and biological properties of membrane vesicles produced by the swine pathogen Streptococcus suis. PLoS One 2015 https://doi.org/10.1371/journal.pone.0130528

This study provides evidence that $S$. suis MVs may play a role as a virulence factor in the pathogenesis of $S$. suis infections, and underlines their excellent potential to be developed as effective acellular vaccine to prevent infections from this pathogen.

15. MacDonald IA, Kuehn MJ: Stress-induced outer membrane vesicle production by Pseudomonas aeruginosa. J. Bacteriol. 2013, 195:2971-2981. 
Resch U, Tsatsaronis JA, Le Rhun A, Stübiger G, Rohde M, Kasvandik S, Holzmeister S, Tinnefeld P, Wai SN, Charpentier E: A two-component regulatory system impacts extracellular membrane-derived vesicle production in group A streptococcus. MBio 2016 https://doi.org/10.1128/mBio.00207-16

17. Lee JH, Choi C-W, Lee T, Kim S Il, Lee J-C, Shin J-H: Transcription factor $\boldsymbol{\sigma}^{\mathbf{B}}$ plays an important role in the production of extracellular membrane-derived vesicles in Listeria monocytogenes. PLoS One 2013 https://doi.org/10.1371/journal.pone.0073196

Rivera J, Cordero RJB, Nakouzi AS, Frases S, Nicola A, Casadevall A: Bacillus anthracis produces membrane-derived vesicles containing biologically active toxins. PNAS 2010, 107:19002-19007.

19. van de Waterbeemd B, Zomer G, van den IJssel J, van Keulen L, Eppink MH, van der Ley P, van der Pol LA: Cysteine depletion causes oxidative stress and triggers outer membrane vesicle release by Neisseria meningitidis; implications for vaccine development. PLoS One 2013 https://doi.org/10.1371/journal.pone.0054314

Surve MV, Anil A, Kamath KG, Bhutda S, Sthanam LK, Pradhan A, Srivastava R, Basu B, Dutta S, Sen S, et al.: Membrane vesicles of group B streptococcus disrupt feto-maternal barrier leading to preterm birth. PLoS Pathog. 2016, 12:e1005816.

21. Lee J, Kim SH, Choi DS, Lee JS, Kim DK, Go G, Park SM, Kim SH, Shin JH, Chang CL, et al.: Proteomic analysis of extracellular vesicles derived from Mycobacterium tuberculosis. Proteomics 2015, 15:3331-3337.

Pathirana RD, Kaparakis-Liaskos M: Bacterial membrane vesicles : biogenesis , immune regulation and pathogenesis. Cell. Microbiol. 2016, 18:1518-1524. adaptive immunity. Int. J. Med. Microbiol. 2014, 304:431-443.

Olaya-abril A, Prados-rosales R, Mcconnell MJ, Martín-peña R, González-reyes JA, Jiménez-munguía I, Gómez-gascón L, Fernández J, Luque-garcía JL, García-lidón C, et al.: Characterization of protective extracellular membrane-derived vesicles produced by Streptococcus pneumoniae. J. Proteomics 2014, 106:46-60.

Prados-rosales R, Carreño LJ, Batista-gonzalez A, Baena A, Venkataswamy MM, Xu J, Yu X, Wallstorm G, Magee DM, LaBaer J, et al.: Mycobacterial membrane vesicles administered systemically in mice induce a protective immune response to surface compartments of Mycobacterium tuberculosis. MBio 2014, 5:e01921-14.

Choi SJ, Kim M, Jeon J, Kim OY, Choi Y, Seo J, Hong S-W, Lee W-H, Jeon SG, Gho YS, et al.: Active immunization with extracellular vesicles derived from Staphylococcus aureus effectively protects against staphylococcal lung infections, mainly via Th1 cell- mediated immunity. PLoS One 2015, 10:e136021.

Haurat MF, Elhenawy W, Feldman MF: Prokaryotic membrane vesicles : new insights on biogenesis and biological roles. Biol. Chem. 2015, 396:95-109.

Kim D-K, Lee J, Kim SR, Choi D-S, Yoon YJ, Kim JH, Go G, Nhung D, Hong K, Jang SC, et al.: EVpedia: a community web portal for extracellular vesicles research. Bioinformatics 2015, 31:933-939.

The authors introduce EVpedia, a free web-based database for extracellular vesicle research. The database contains relevent publications, vesicular components and bioinformatic tools, which benefit the emerging field of extracellular vesicle research.

30. Kim D-K, Lee J, Simpson RJ, Lötvall J, Gho YS: EVpedia: a community web resource for prokaryotic and eukaryotic extracellular vesicles research. Semin. Cell Dev. Biol. 2015, 40:4-7.

31. Liao S, Klein MI, Heim KP, Fan Y, Bitoun JP, Ahn S-J, Burne RA, Koo H, Brady LJ, Wen ZT: Streptococcus mutans extracellular DNA is upregulated during growth in biofilms, actively released via membrane vesicles, and influenced by components of the protein secretion machinery. J. Bacteriol. 2014, 196:2355-2366.

32. Soler N, Krupovic M, Marguet E, Forterre P: Membrane vesicles in natural environments: a major challenge in viral ecology. ISME J. 2015, 9:793-796.

Gaudin M, Krupovic M, Marguet E, Gauliard E, Cvirkaite-Krupovic V, Le Cam E, Oberto J, Forterre P: Extracellular membrane vesicles harbouring viral genomes. Environ. Microbiol. 2014, 16:1167-1175.

Sjöström AE, Sandblad L, Uhlin BE, Wai SN: Membrane vesicle-mediated release of bacterial RNA. Sci. Rep. 2015 , 5:15329.

Fulsundar S, Harms K, Flaten GE, Johnsen PJ, Chopade BA, Nielsen KM: Gene transfer potential of outer membrane vesicles of Acinetobacter baylyi and effects of stress on vesiculation. Appl. Environ. Microbiol. 2014, 80:3469-3483. 38:16-21.

37. Wang L, Li F, Dang L, Liang $\mathrm{C}$, Wang $\mathrm{C}$, He B, Liu J, Li D, Wu X, Xu X, et al.: In vivo delivery systems for therapeutic genome editing. Int. J. Mol. Sci. 2016 https://doi.org/10.3390/ijms17050626 

genetically modified organisms and repealing Council Directive 90/220/EEC. Off. J. Eur. Communities 2001, L 106/1:1-38.

39. Vasilyeva N V., Tsfasman IM, Kudryakova I V., Suzina NE, Shishkova NA, Kulaev IS, Stepnaya OA: The role of membrane vesicles in secretion of Lysobacter sp. bacteriolytic enzymes. J. Mol. Microbiol. Biotechnol. 2013, 23:142-151.

40. Schrempf H, Merling P: Extracellular Streptomyces lividans vesicles: composition, biogenesis and antimicrobial activity. Microb. Biotechnol. 2015, 8:644-658.

41. Kim Y, Edwards N, Fenselau C: Extracellular vesicle proteomes reflect developmental phases of Bacillus subtilis. Clin. Proteomics 2016, 13. https://doi.org/10.1186/s12014-016-9107-z

Schrempf H, Koebsch I, Walter S, Engelhardt H, Meschke H: Extracellular Streptomyces vesicles: amphorae for survival and defence. Microb. Biotechnol. 2011, 4:286-299.

Jeon J, Mok HJ, Choi Y, Park SC, Jo H, Her J, Han J-K, Kim Y-K, Kim KP, Ban C: Proteomic analysis of extracellular vesicles derived from Propionibacterium acnes. PROTEOMICS - Clin. Appl. 2017 https://doi.org/10.1002/prca.201600040

44. Lee J, Lee E-Y, Kim S-H, Kim D-K, Park K-S, Kim KP, Kim Y-K, Roh T-Y, Gho YS: Staphylococcus aureus extracellular vesicles carry biologically active $\beta$-lactamase. Antimicrob. Agents Chemother. 2013, 57:2589-2595.

Rezaïki L, Lamberet G, Derré A, Gruss A, Gaudu P: Lactococcus lactis produces short-chain quinones that cross-feed group B streptococcus to activate respiration growth. Mol. Microbiol. 2008, 67:947-957.

Das A, Hugenholtz J, Van Halbeek H, Ljungdahl LG: Structure and function of a menaquinone involved in electron transport in membranes of Clostridium thermoautotrophicum and Clostridium thermoaceticum. J Bacteriol 1989, 171:5823-5829.

47. Erkus O, de Jager VC, Spus M, van Alen-Boerrigter IJ, van Rijswijck IM, Hazelwood L, Janssen PW, van Hijum SA, Kleerebezem M, Smid EJ: Multifactorial diversity sustains microbial community stability. ISME J. 2013, 7:2126-2136.

Hardin G: The competitive exclusion principle. Science. 1960, 131:1292-1297. 1517.

51. Vermeer C, Schurgers LJ: A comprehensive review of vitamin K and vitamin K antagonists. Hematol. Oncol. Clin. North Am. 2000, 14:339-353. 1903. increase production ratio of 1,4-dihydroxy-2-naphthoic acid to menaquinone. J. Biosci. Bioeng. 2006, 101:464-470. https://doi.org/10.1038/ncomms10515 